\title{
Optimal management of muscle-invasive bladder cancer - a review
}

\author{
This article was published in the following Dove Press journal: \\ Research and Reports in Urology \\ 4 September 2015 \\ Number of times this article has been viewed
}

\author{
Kristen R Scarpato' \\ Alicia K Morgans ${ }^{2}$ \\ Kelvin A Moses' \\ 'Department of Urologic Surgery, \\ 2Division of Hematology/Oncology, \\ Vanderbilt University Medical Center, \\ Nashville, TN, USA
}

\begin{abstract}
Muscle-invasive bladder cancer is a complex disease requiring aggressive management. Patients are often older with comorbid conditions that impact treatment options. This review describes the available therapies for invasive urothelial carcinoma, including chemotherapy, radical extirpative surgery, and bladder-preserving strategies.
\end{abstract}

Keywords: bladder cancer, urothelial carcinoma, neoadjuvant chemotherapy, radical cystectomy

\section{Introduction}

Bladder cancer is a heterogeneous disease that will account for an estimated 74,000 new cases and 16,000 deaths in the USA in $2015 .{ }^{1}$ Approximately $75 \%$ of patients will present with noninvasive bladder cancer, but nearly a quarter have muscle-invasive disease at the time of diagnosis. ${ }^{2}$ Importantly, no standard method of providing optimal-quality care for muscle-invasive disease has been widely adopted, and treatment algorithms for muscle-invasive bladder cancer (MIBC) vary significantly by institution. Despite therapeutic advancements, there remains significant room for improvement in providing consistent, evidence-based, and high-quality $\mathrm{BC}$ care. ${ }^{3}$

\section{Standard treatment}

While patients with NMI urothelial carcinoma can often be safely managed with transurethral resection with or without intravesical chemotherapy or immunotherapy, neoadjuvant chemotherapy followed by radical cystectomy (RC) with bilateral pelvic lymph-node dissection (PLND) is the standard of care for patients with MIBC.,5 The goal of RC is to remove all of the cancer within the bladder and surrounding tissues and to achieve negative margins. Concomitant thorough PLND is both diagnostic and therapeutic, with a significant increase in overall survival (OS) associated with PLND in nonmetastatic patients across all tumor stages. ${ }^{6}$

\section{Timely diagnosis and treatment}

Due to the aggressive nature of the disease, timely diagnosis and prompt surgical treatment are keys in the management of MIBC. Delay in diagnosis or cystectomy adversely impacts outcomes. ${ }^{7,8}$ Using Surveillance, Epidemiology, and End Results (SEER)-Medicare data to assess time between initial hematuria evaluation and bladder cancer diagnosis, Hollenbeck et al found that delays in bladder cancer diagnosis significantly increased disease specific mortality (hazard ratio [HR] 1.34, 95\%
Correspondence: Kelvin A Moses
Department of Urologic Surgery, Vanderbilt University Medical Center, 11612 Ist Avenue South - MCN A-1302, Nashville, TN 37232, USA Tel + I 6153431317

Fax + I 6153228990

Email kelvin.a.moses@vanderbilt.edu submit your manuscript $\mid$ www.dovepress.com

Dovepress

http://dx.doi.org/1 0.2147/RRU.S73566 
confidence interval [CI] 1.20-1.50). ${ }^{7}$ Patients with longer delays to treatment tended to have a greater number of comorbid conditions, were older, and were male. Delay in diagnosis had a greater negative impact on bladder cancer outcomes than grade or stage. Similarly, Garg et al found that women experienced longer mean time to urologic follow-up for hematuria, with subsequently delayed hematuria evaluation. ${ }^{9}$ These delays may account for existing sex disparities in bladder cancer outcomes, with females presenting at later stages and experiencing poorer diseasespecific survival (DSS). Using results from a SEER analysis of 441 patients diagnosed with MIBC, Gore et al demonstrated that delay in time to definitive surgical treatment beyond 12 weeks resulted in increased disease-specific mortality (HR 2.0, $P<0.01$ ) and overall mortality (HR 1.6, $P<0.01) .{ }^{8}$ Reasons for treatment delay were often related to patient comorbidities and reluctance to undergo radical surgery with urinary diversion (UD).

\section{Preoperative assessment and management}

For those who receive neoadjuvant chemotherapy, more than $60 \%$ of patients will have residual disease justifying subsequent consolidative radical therapy.$^{10}$ In these patients, or those undergoing upfront $\mathrm{RC}$, a thorough metastatic workup and medical evaluation considering age, nutrition, and performance status are necessary.

\section{Metastatic evaluation}

For patients found to have T2 disease on transurethral resection, staging studies of the chest, abdomen, and pelvis, including upper-tract evaluation, must be performed to rule out metastases, given the significant potential for nodal disease. Detecting metastatic nodes can alter treatment decisions and result in avoidance of unnecessary radical surgery in some patients, while encouraging the use of neoadjuvant chemotherapy in others. Currently, the gold standard for staging in bladder cancer is computed tomography (CT), although more recently multiparametric magnetic resonance imaging and positron-emission tomography (PET) imaging have shown promise. ${ }^{11-13}$ Utilization of non- ${ }^{18} \mathrm{~F}$-fluorodeoxyglucose (FDG) PET can allow for detection of metastatic disease without signal interference caused by concentration of the metabolite in urine. In a publication from Italy ${ }^{11} \mathrm{C}$-choline-PET CT was utilized preoperatively for disease staging and postoperatively to disease relapse. ${ }^{11}$ Overall, ${ }^{11} \mathrm{C}$-choline-PET CT detected $63 \%$ of metastatic nodes with a sensitivity of $59 \%$ and specificity of $90 \%$ when compared with histologic specimens. Goodfellow et al evaluated $233 \mathrm{RC}$ candidates with CT scans of the chest, abdomen, and pelvis, as well as with FDG PET. ${ }^{12}$ Imaging findings were correlated with pathology specimens, resulting in an FDG PET sensitivity of 54\% for disease outside the pelvis versus $41 \%$ with CT scan, with similar specificities of $97 \%$ and $98 \%$, respectively. The authors concluded that while combined FDG PET and CT slightly improved detection of metastatic disease, it should only be considered in select patients, given its significant cost.

\section{Nutrition}

$\mathrm{RC}$ is a morbid procedure that necessitates preoperative medical optimization. An important part of surgical preparation and postoperative management is attaining good nutritional status, which is thought to significantly impact patient outcomes. In a retrospective review of a National Surgical Quality Improvement Program database, Johnson et al evaluated the role of preoperative nutritional status, as measured by serum albumin, in postsurgical complications. ${ }^{14}$ In over 1,200 patients who underwent $\mathrm{RC}$, preoperative albumin $<3.5 \mathrm{gm} / \mathrm{dL}$ was the only significant predictor of postoperative complication $(P=0.03)$. This cohort of patients had twice the risk of 30-day complications when compared to patients with normal serum albumin. Preoperative nutrition has also been shown to impact mortality following cystectomy. ${ }^{15}$ Parameters including albumin $<3.5 \mathrm{gm} / \mathrm{dL}$, body mass index $<18.5 \mathrm{~kg} / \mathrm{m}^{2}$, and preoperative weight loss $>5 \%$ of body weight were used as indicators of nutritional deficiency. Nearly $20 \%$ of the study population was nutritionally deficient, which translated into higher 90-day mortality rates $(16.5 \%$ versus $5.1 \%, P<0.01)$ and decreased OS at 3 years $(44.5 \%$ versus $67.6 \%, P<0.01)$. Both of these studies highlight the need for nutritional assessment and optimization to improve RC outcomes, and prospective studies are ongoing.

\section{Age}

Bladder cancer primarily affects older patients, and as the population ages, urologists are increasingly faced with elderly, comorbid patients needing treatment for IBC. Given the morbid nature of $\mathrm{RC}$, there is concern regarding the impact of advanced age on postoperative morbidity and mortality. Schiffmann et al examined 30-day and 90-day postoperative mortality rates and evaluated the impact of age, socioeconomic status, and comorbid conditions. ${ }^{16}$ Utilizing a SEER-Medicare linked database, 5,207 patients with nonmetastatic MIBC were identified and found to be increasingly 
older and sicker over the study period: 1991-2009. Mortality was greater at 90 days $(10.6 \%)$ than at 30 days $(5.2 \%)$, with increasing 90-day mortality rates according to age and comorbidity index. Despite this, the study demonstrated stable 90-day mortality rates over time, pointing to improved outcomes, perhaps due to enhanced patient care or better patient selection. Contrary to this, Donat et al found that $\mathrm{RC}$ in octogenarians provided a nonsignificantly higher rate of both major and minor complications $(P=0.15)$, while providing similar disease control and DSS when compared to those patients younger than 80 years. ${ }^{17}$ Until a clear age cut point is established, RC should remain the standard of care for MIBC, and elderly patients should receive equally aggressive therapy.

\section{Frailty and performance status}

As previously mentioned cystectomy candidates are often older patients with nutritional deficiency and increased comorbidity. Other parameters, such as frailty and performance status, have been developed to increase objectivity and aid in determining which patients are appropriate for surgery.

Surgeons have progressively adopted frailty-assessment tools using well-established methodologies as a predictive measure in the geriatric population. Revenig et al ${ }^{18}$ prospectively evaluated 30-day postoperative complications in 189 patients undergoing major abdominal surgery (including 117 urologic patients) using the validated Hopkins Frailty Score, self-administered questionnaires, performance status, and laboratory values, and found that patients who were intermediately frail or frail were more likely to experience complications (odds ratio $2.07,95 \%$ CI $1.05-4.08$ ). Urologists should consider a patient's ability to tolerate this potentially morbid procedure, and frailty has been shown to correlate with fitness for surgery.

Another predictor of outcomes following surgery is the Karnofsky Performance Status (KPS) scale. Evers et al performed a retrospective analysis on $234 \mathrm{RC}$ patients and evaluated the relationship of variables including KPS, the Charlson Comorbidity Index (CCI), and American Society of Anesthesiologists (ASA) class with OS, cancer-specific survival (CSS), and progression-free survival. ${ }^{19} \mathrm{KPS}$ was found to be an independent predictor of OS, with a score $\leq 80$ correlating with decreased survival, as well as CSS and progression-free survival, while the CCI and ASA class were not predictive. Similarly, a group from the Mayo Clinic evaluated perioperative and 5-year all-cause mortality following $\mathrm{RC}$ in relationship to patient comorbidity indices, including ASA class, CCI, Elixhauser Index (EI), and Eastern Cooperative Oncology Group (ECOG) performance status. ${ }^{20}$ On multivariate analysis, ASA class (HR 3.17, $P=0.001$ ), EI (HR 1.48, $P=0.002$ ), and ECOG performance status (HR $2.4, P<0.0001)$ significantly impacted 90 -day perioperative mortality. Furthermore, all indices were independently associated with 5-year all-cause mortality, leading the authors to conclude that comorbidity status is an essential consideration in patient counseling, outcomes research, and quality improvement. The ideal prognostic comorbidity index remains elusive. In a retrospective multicenter analysis, Mayr et al found that comorbidity status, as defined by Adult Comorbidity Evaluation 27, CCI, age-adjusted CCI, ECOG performance status, and ASA score, was predictive of cancer-independent mortality in patients with urothelial carcinoma undergoing cystectomy, but did not correlate with cancer-specific mortality. ${ }^{21}$

\section{Surgery for MIBC}

$\mathrm{RC}$ with lymph-node dissection remains the standard treatment for patients with MIBC or high-risk NMIBC. Existing guidelines and practice statements dedicated to preventing perioperative complications like infection and venous thromboembolism (VTE) help guide perioperative management. Based on patient and procedural factors, as well as the potential morbidity of infection, the American Urological Association advocates the use of a second- or third-generation cephalosporin or a combination of an aminoglycoside with metronidazole or clindamycin for a maximum of 24 hours for surgery involving the intestine. ${ }^{22} \mathrm{~A}$ preoperative dose of Lovenox or heparin is given, and compression stockings placed prior to the procedure.

Creation of a UD following bladder removal necessitates manipulation of the bowel, and historically preoperative bowel preparation has been utilized. Zaid et al reviewed the role of bowel preparation preceding cystectomy, and found level I evidence did not support the routine use of mechanical bowel preparation with agents like polyethylene glycol or oral sodium phosphate. ${ }^{23}$

\section{Open versus robotic $\mathrm{RC}$}

Controversy with regard to cystectomy has focused on the best surgical approach (open versus robotic), and the optimal extent of lymph-node dissection. As urologists become more facile with robotic-assisted procedures, many centers have adopted robot-assisted RC (RARC). There is much debate in the literature comparing open $\mathrm{RC}$ to RARC, evaluating outcomes, complications, and cost. Bochner et al compared 
the two techniques in a randomized control trial of 118 patients, in which they considered 90-day complications utilizing the Clavien system, as well as compared highgrade complications, estimated blood loss, operative time, pathologic outcomes, quality of life, and cost. ${ }^{24}$ Overall, the complication rates were similar between the robotic group and the open cohort ( $62 \%$ versus $66 \%, P=0.7$ ), and the study was closed early for futility at the interim analysis. Early results showed lower estimated blood loss $(P=0.027)$ for the robotic cohort, but longer operative times $(P<0.001)$ and higher cost $(P<0.05)$ than those who underwent open $\mathrm{RC}$. There was no difference in margin status, lymph-node yield, length of hospital stay, or quality of life. Therefore, the authors concluded that there was no significant patient benefit to RARC compared to open surgery.

In a systematic literature review, Yuh et al examined oncologic and functional outcomes following RARC. ${ }^{25}$ They did not find an association between lymph-node yield, positive margin status, disease-free survival, CSS, or OS between RARC and open RC, although limited oncologic outcome data were available for review. Data on functional outcomes were also sparse, with daytime continence rates ranging from $83 \%$ to $100 \%$ and nighttime $66 \%$ to $76 \%$ among patients who had orthotopic diversion. These early data point to comparable outcomes between the gold-standard open $\mathrm{RC}$ and RARC. Using a population-based analysis, Leow et al compared morbidity and cost of open RC and RARC. ${ }^{26}$ Rates of major complications were similar between the two, but RARC was associated with decreased chance of minor complications $(P=0.03)$ and was significantly more expensive due to supply costs $(P<0.0001)$. With oncologic outcomes being the primary objective of $\mathrm{RC}$, newer research has evaluated patterns of recurrence following open and robotic RC. In a retrospective comparison of 383 patients, Nguyen et $\mathrm{al}^{27}$ found variable patterns of distant recurrence, with more frequent extrapelvic lymph-node location peritoneal carcinomatosis in the RARC patients, although there was no difference in rates of local or distant recurrence overall. It is hypothesized that pneumoperitoneum may facilitate seeding of bladder cancer cells.

In some high-volume centers, surgeons have been performing intracorporeal UD (ICUD) following RARC. Ahmed et al reviewed the database of the International Robotic Cystectomy Consortium, and compared outcomes between ICUD and extracorporeal UD following RARC. ${ }^{28}$ Nearly $20 \%$ of the 935 patients who had RARC underwent ICUD. The authors found equivalent operative times and an insignificantly longer median hospital stay for ICUD. There was no difference in reoperation or complication rate at 30 days or 90 days, although fewer 30-day $(P<0.001)$ and 90 -day $(P=0.016)$ readmissions and gastrointestinal complications $(P \leq 0.001)$ were seen in the ICUD group. ICUD was found to be technically feasible, with improved postoperative parameters.

\section{Lymphadenectomy}

Lymphadenectomy is considered both diagnostic and therapeutic. However, controversy regarding optimal number of nodes or extent of lymph-node dissection (LND) at the time of RC persists. In a comparative study of superextended versus extended pelvic LND, extended intrapelvic template dissection boundaries were the circumflex iliac vein and Cloquet's node distally, the pelvic side wall and genitofemoral nerve laterally, the obturator nerve posteriorly, and the mid-upper third of the common iliac vessels superiorly. ${ }^{29}$ The superextended dissection included lymph-node tissue up to the inferior mesenteric artery takeoff, as well as complete dissection of the presacral space. No difference in recurrence-free survival or recurrence rate was seen for the variable dissection templates. Stein et al demonstrated the importance of performing a thorough and meticulous LND, and showed the number of positive lymph nodes impacted prognosis. ${ }^{5}$ They found that fewer than five positive nodes resulted in 10-year recurrence-free survival of $41 \%$ versus $24 \%$ in patients with greater than five positive nodes. In a recent PubMed review, Morgan et al found that disagreement about management of regional lymph nodes persists, although there is strong evidence in support of extended LND. ${ }^{30}$ Using a SEER-Medicare linked database, Morgan et al found that in node-negative patients, lymph-node count was associated with OS and DSS, but that relationship did not exist in node-positive patients. ${ }^{31}$ For node-negative patients with $\leq 5$ nodes removed, the risk of mortality was significantly greater than for patients with $\geq 14$ nodes removed, possibly due to improved staging, removal of any micrometastatic disease, or perhaps improved patient parameters and surgeon expertise.

A systematic PubMed search, summarizing data on the impact of lymphadenectomy for staging and bladder cancer outcomes found that quality, prospective data are limited. ${ }^{32}$ Existent data indicate that the extent of LND impacts DSS, regardless of LN positivity and cancer stage. Until prospective high-quality data exist, the benefits of extended lymphadenectomy must be weighed against potential complications, including bleeding, nerve injury, lymphocele, and lower-extremity thrombus. The Southwest Oncology Group is currently conducting a Phase III trial (SWOG S1011) to 
evaluate standard and extended pelvic lymphadenectomy in patients undergoing $\mathrm{RC}$ for MIBC to help establish which may be more effective.

\section{Postoperative management}

The development of postoperative care pathways that largely focus on avoidance of bowel complications has been shown to improve surgical outcomes and enhance recovery following cystectomy. As previously mentioned, level I evidence does not support the routine use of mechanical bowel preparation with agents like polyethylene glycol or oral sodium phosphate. ${ }^{23}$ Postoperatively, newer evidence supports the omission of nasogastric tube decompression while instituting early feeding and bowel regimens to facilitate return of bowel function and thus earlier hospital discharge. More recently, the use of $\mu$-opioid-receptor antagonists have been shown to limit postoperative ileus. In a multicenter randomized controlled trial, Lee et al found that alvimopan enhanced bowel recovery postcystectomy, shortening the length of stay (LOS) without significantly increasing side effects. ${ }^{33}$ Patients randomized to alvimopan were able to tolerate solid food and have a bowel movement 1.3 days sooner than those who received placebo $(P<0.0001)$. Kauf et al demonstrated that cystectomy patients who received alvimopan had an average 2.63-day shorter LOS, with lower postoperative costs related to ileus. ${ }^{34}$ The University of Southern California initiated a perioperative protocol in 110 open RC patients to enhance recovery. The protocol avoided bowel preparation and nasogastric tube decompression, minimized narcotic pain-relief use, instituted early feeding, and employed cholinergic and $\mu$-opioid antagonists. ${ }^{35}$ This cohort experienced a median LOS of 4 days, compared to nonprotocol patients at 8 days, with the majority $(82 \%)$ having return of bowel function on postoperative day 2 . There was no increase in hospital readmission rates.

In 2008, the American Urological Association published a best-practice statement for the prevention of deep-vein thrombosis for urologic patients. ${ }^{36}$ In patients undergoing laparoscopic or robotic cystectomy, sequential compression devices are recommended, while patients considered at high risk based on history and comorbidities should receive low-molecular-weight heparin. Data supporting these recommendations are lacking. Patients undergoing open procedures are felt to be at greater risk, and more aggressive prophylaxis with sequential compression devices and low-molecular-weight heparin is recommended. Sun et al sought to further elucidate risk factors for VTE in patients following open $\mathrm{RC}$ for bladder cancer in a retrospective review of 2,316 patients. $^{37}$ Symptomatic VTE was diagnosed in $4.7 \%$ of patients, over half of whom presented after discharge home. Development of VTE was significantly related to body mass index, surgical margin status, type of UD, and LOS. Use of pharmacologic prophylaxis was not a significant predictor of VTE.

\section{Perioperative chemotherapy Neoadjuvant chemotherapy}

Prior to the development of effective combination chemotherapy, patients experienced high relapse rates within 2 years of radical local therapy, likely due to micrometastatic disease. The development of effective perioperative chemotherapy has positively impacted disease outcomes for MIBC..$^{10,38-40}$ The most commonly used neoadjuvant regimens are cisplatinbased combinations, including methotrexate, vinblastine, doxorubicin, and cisplatin (MVAC), gemcitabine plus cisplatin (GC), and cisplatin, methotrexate, and vinblastine.

Several studies have demonstrated that treatment of patients with MIBC with neoadjuvant chemotherapy prior to RC improves survival. The first was an 11-year Southwest Oncology Group trial comparing neoadjuvant MVAC and $\mathrm{RC}$ to cystectomy alone in patients with locally advanced bladder cancer. ${ }^{10}$ Median survival in patients who received neoadjuvant MVAC was 77 months versus 46 months in those who underwent cystectomy alone $(P=0.06)$, with the cystectomy patients having a 33\% higher risk of death than those receiving combination therapy (HR 1.33, 95\% CI 1.00-1.76). In subgroup analyses, median survival was improved for patients aged $<65$ years $(P=0.05)$ and the survival benefit held across tumor stages, including for patients with pathologic T2 disease or lower $(P=0.05)$. Additionally, neoadjuvant chemotherapy resulted in significantly greater downstaging of disease, with $38 \%$ of patients achieving pT0 at the time of cystectomy compared with only $15 \%$ of those who underwent initial transurethral resection of bladder tumor (TURBT) and cystectomy alone. This is particularly important, because achieving a pathologic complete response (pT0N0M0) is associated with a $55 \%$ lower risk of death and an $81 \%$ lower risk of recurrence. ${ }^{41}$

Additional evidence suggests a benefit from treatment with neoadjuvant chemotherapy. The long-term results of an international multicenter randomized controlled trial comparing neoadjuvant cisplatin, methotrexate, and vinblastine to local radical treatment (cystectomy or radiotherapy) alone in patients with locally advanced disease demonstrated a $16 \%$ reduction in the risk of death $(P=0.037)$ and improved metastasis-free survival $(P=0.001)$ and disease-free survival 
$(P=0.008) .{ }^{39}$ Survival increased from $50 \%$ to $56 \%$ at 3 years and from $30 \%$ to $36 \%$ at 10 years. Further, results of the Advanced Bladder Cancer Meta-analysis Collaboration, including eleven trials and 3,005 patients, suggests a $5 \%$ significant survival benefit associated with platinumbased neoadjuvant chemotherapy at 5 years (HR 0.86, 95\% CI $0.77-0.95 ; P=0.003) .{ }^{42}$ Based on these data, the National Comprehensive Cancer Network in the USA and the Second International Consultation on Bladder Cancer recommend neoadjuvant chemotherapy for patients with MIBC to improve survival and downstage pathology. ${ }^{4,40}$ Despite the evidence in support of perioperative chemotherapy in the treatment of MIBC, uptake in the community remains suboptimal. ${ }^{43}$

Controversy exists regarding which neoadjuvant chemotherapy combination is superior. Many institutions opt to use GC rather than MVAC, due to evidence gathered from a randomized controlled trial of patients with locally advanced or metastatic bladder cancer who did not undergo RC. Investigators randomized 405 patients with advanced bladder cancer not receiving RC to GC or standard MVAC, and found similar OS, disease progression, time to treatment failure, and response rates. ${ }^{38} \mathrm{GC}$ had a better safety and tolerability profile in this population, with lower rates of neutropenia, mucositis, and alopecia compared to standard MVAC. Many physicians extrapolate this superior safety and tolerability data to the neoadjuvant setting, and use GC rather than MVAC as the preferred neoadjuvant regimen, although these results have not been replicated in the neoadjuvant setting. Galksy et al further explored this issue in a recent retrospective large electronic data-capture study evaluating pathologic complete response in patients who had received MVAC $(77 \%$ received dose-dense and $23 \%$ received standard dose) or GC. ${ }^{44}$ Of the 212 patients who met the criteria, $29 \%$ versus $31 \%$ achieved pathologic response in the MVAC and GC cohorts, respectively $(P=0.77)$. Similarly, an exploratory analysis of survival in the two cohorts found no significant difference $(P=0.48)$.

Dose-dense MVAC has emerged as another viable regimen for patients. The European Organisation for Research and Treatment of Cancer protocol 30924 compared standard MVAC to dose-dense MVAC plus granulocyte colony-stimulating factor in patients with advanced disease, and found significantly better response rates for the dose-dense regimen $(P=0.016)$, with $25 \%$ having a complete response versus only $11 \%$ in the classic MVAC arm $(P=0.006) .{ }^{45}$ The improved response coupled with the shorter delivery time and improved toxicity has led to dose-dense MVAC use in the neoadjuvant setting.

\section{Adjuvant chemotherapy}

According to National Comprehensive Cancer Network guidelines, patients who have not received neoadjuvant chemotherapy and have pT3-4 disease or positive nodes following RC with LND should consider adjuvant chemotherapy. ${ }^{4}$ Sternberg et al similarly recommended cisplatin-based therapy for patients who have not received neoadjuvant therapy and have extravesical extension or regional lymph-node metastasis, although the authors noted that chemotherapy may be difficult to administer following $\mathrm{RC}$ and sufficient evidence of benefit is lacking. ${ }^{40}$

A large multi-institutional study retrospectively reviewed over 1,500 patients with lymph node-positive disease following RC, and found that cisplatin-based adjuvant chemotherapy administered within 3 months of surgery was associated with an $18 \%$ relative risk reduction in diseasespecific mortality $(P=0.005)$, with an absolute reduction in mortality of $3.5 \%$ at 5 years. ${ }^{46}$ The benefit of adjuvant chemotherapy was most pronounced in patients who were younger, female, and had pT3-4 disease, high lymph-node density, and lymphovascular invasion. A recent randomized Phase III trial evaluated immediate versus deferred adjuvant chemotherapy for patients with pT3-4 or $\mathrm{N}^{+} \mathrm{M} 0$ bladder cancer. ${ }^{47}$ Patients received either immediate MVAC or GC, or deferred chemotherapy at relapse. There was no difference seen in OS, although immediate chemotherapy significantly prolonged progression-free survival. However, the study was closed due to low patient accrual, limiting its power to detect significant differences. More prospective studies regarding the timing of adjuvant chemotherapy are needed to clarify the remaining uncertainty.

\section{Bladder-preservation strategies}

Although not the standard of care, at most US institutions, bladder-preserving treatments continued to evolve for patients refusing or not considered candidates for RC. Arcangeli et al performed a systematic review and metaanalysis of clinical trials evaluating trimodality therapy utilizing transurethral resection followed by chemotherapy and radiation for MIBC. ${ }^{48}$ They found that $78 \%$ of patients had a complete response, with 5-year OS of 56\%. Patients who received continuous treatment rather than split therapy have a higher likelihood of complete response and lower salvage cystectomy rates. Results regarding late toxicity of bladderpreserving strategies are still lacking. A pooled analysis of multiple Radiation Therapy Oncology Group protocols with long-term outcomes of bladder preservation therapy using TURBT and radiation with concurrent chemotherapy (all 
of which were cisplatin-based) was published in $2014 .{ }^{49} \mathrm{~A}$ complete response was seen in $69 \%$ of patients, with 5 - and 10 -year survival of $57 \%$ and $36 \%$, respectively, comparable to immediate cystectomy in some studies. Additionally, patients who did require salvage cystectomy had a 5 -year DSS of $60 \%$ and 10 -year DSS of $47 \%$.

James et al performed a multicenter randomized control trial evaluating radiotherapy and radiotherapy combined with radiosensitizing agents, fluorouracil, and mitomycin $\mathrm{C}$ in patients with T2-T4aN0M0 disease and good performance status. ${ }^{50}$ The addition of chemotherapy to radiation improved local control (relative reduction of recurrence of $33 \%$, $P=0.03$ ) and decreased invasive local recurrence by nearly $50 \%$ when compared to radiotherapy alone. In this study, synchronous chemoradiation resulted in reasonable local control and maintenance of a functional bladder while avoiding the use of cisplatin-based therapy, which is often not an option for patients who have renal insufficiency and are too ill to undergo cystectomy. Another potential bladder-preservation modality is intra-arterial chemotherapy combined with radiotherapy. ${ }^{51}$ Although data are limited, intra-arterial chemotherapy, which may be given via the femoral artery and directed at the tumor blood supply, given in combination with radiation can result in pathologic complete response rates of $43 \%$ and 5-year OS rates of 50.3\%. More research is needed in this area. Recent data from Japan evaluated the role of balloon-occluded arterial infusion of cisplatin and gemcitabine given in conjunction with hemodialysis and radiation in patients with advanced bladder cancer, and showed promising initial results. ${ }^{52}$

\section{Future directions and addressing disparities}

Significant improvement is needed to address the disparities in outcomes among patients with MIBC. Although bladder cancer is less common in females than in males, ${ }^{1}$ women typically present with more advanced disease and experience worse outcomes. A recent population-based analysis using a SEER database highlighted the sex disparity in hematuria evaluation and bladder cancer diagnosis. ${ }^{9}$ Results showed longer time to urologic consultation for women, as well as a significant delay in initial hematuria evaluation.

Despite recent advances and continuously declining death rates, racial disparity also persists. Blacks experience $13 \%$ lower 5-year survival rates than whites for all stages of bladder cancer, thought to be attributed to delays in diagnosis, patient comorbidities, and variable treatments. ${ }^{53}$ Trends in 5-year survival from 1975 to 2010 indicated that survival rates increased from $50 \%$ to $64 \%$ for blacks compared to $73 \%$ to $80 \%$ for whites. Additionally, black patients more commonly present with regional or distant disease than nonblacks. ${ }^{1}$

Emerging data regarding health literacy suggests that the ability to obtain and act upon surgical information impacts outcomes. Treatment for MIBC involves a complex series of therapies and a potentially morbid surgery that may require intensive postoperative self-care. Addressing health literacy to improve patient and caregiver understanding of what is required to achieve best outcomes may lead to a reduction in complications and lower readmission rates.

There remains a critical need to improve bladder cancer outcomes across all socioeconomic, racial, and educational cohorts. Future clinical trials and quality-of-care initiatives focused on these disparities are necessary to improve outcomes for all. This includes adding to the paucity of data on health-related quality of life and cancer survivorship following treatment for MIBC.

\section{Conclusion}

MIBC requires intensive management, and results in substantial health care expenditure. While progress has been made, more prospective studies that meet accrual goals are needed to optimize management and improve outcomes for all patients.

\section{Disclosure}

The authors report no conflicts of interest in this work.

\section{References}

1. Siegel RL, Miller KD, Jemal A. Cancer statistics, 2015. CA Cancer J Clin. 2015;65:5-29.

2. Amin MB, McKenney JK, Paner GP, et al. ICUD-EAU International Consultation on Bladder Cancer 2012: pathology. Eur Urol. 2013;63: $16-35$.

3. Chamie K, Litwin MS. Quality of bladder cancer care in the USA. Expert Rev Pharmacoecon Outcomes Res. 2011;11:619-621.

4. National Comprehensive Cancer Network. NCCN Guidelines Version 1.2015. 2015. Available from: http://www.nccn.org/professionals/ physician_gls/PDF/bladder.pdf. Accessed April 30, 2015.

5. Stein JP, Lieskovsky G, Cote R, et al. Radical cystectomy in the treatment of invasive bladder cancer: long-term results in 1,054 patients. J Clin Oncol. 2001;19:666-675.

6. Abdollah F, Sun M, Schmitges J, et al. Stage-specific impact of pelvic lymph node dissection on survival in patients with non-metastatic bladder cancer treated with radical cystectomy. BJU Int. 2012;109:1147-1154.

7. Hollenbeck BK, Dunn RL, Ye Z, et al. Delays in diagnosis and bladder cancer mortality. Cancer. 2010;116:5235-5242.

8. Gore JL, Lai J, Setodji CM, Litwin MS, Saigal CS. Mortality increases when radical cystectomy is delayed more than 12 weeks: results from a Surveillance, Epidemiology, and End Results-Medicare analysis. Cancer. 2009;115:988-996.

9. Garg T, Pinheiro LC, Atoria CL, et al. Gender disparities in hematuria evaluation and bladder cancer diagnosis: a population based analysis. $J$ Urol. 2014;192:1072-1077. 
10. Grossman HB, Natale RB, Tangen CM, et al. Neoadjuvant chemotherapy plus cystectomy compared with cystectomy alone for locally advanced bladder cancer. $N$ Engl J Med. 2003;349:859-866.

11. Ceci F, Bianchi L, Graziani T, et al. 11C-choline PET/CT and bladder cancer: lymph node metastasis assessment with pathological specimens as a reference standard. Clin Nucl Med. 2015;40:e124-e128.

12. Goodfellow H, Viney Z, Hughes P, et al. Role of fluorodeoxyglucose positron emission tomography (FDG PET)-computed tomography (CT) in the staging of bladder cancer. BJU Int. 2014;114:389-395.

13. McKibben MJ, Woods ME. Preoperative imaging for staging bladder cancer. Curr Urol Rep. 2015;16:22.

14. Johnson DC, Riggs SB, Nielsen ME, et al. Nutritional predictors of complications following radical cystectomy. World J Urol. 2015;33: $1129-1137$.

15. Gregg JR, Cookson MS, Phillips S, et al. Effect of preoperative nutritional deficiency on mortality after radical cystectomy for bladder cancer. J Urol. 2011;185:90-96.

16. Schiffmann J, Gandaglia G, Larcher A, et al. Contemporary 90-day mortality rates after radical cystectomy in the elderly. Eur J Surg Oncol. 2014;40:1738-1745.

17. Donat SM, Siegrist T, Cronin A, Savage C, Milowsky MI, Herr HW. Radical cystectomy in octogenarians - does morbidity outweigh the potential survival benefits? J Urol. 2010;183:2171-2177.

18. Revenig LM, Canter DJ, Taylor MD, et al. Too frail for surgery? Initial results of a large multidisciplinary prospective study examining preoperative variables predictive of poor surgical outcomes. $J$ Am Coll Surg. 2013;217:665-670. e1.

19. Evers PD, Logan JE, Sills V, Chin AL. Karnofsky Performance Status predicts overall survival, cancer-specific survival and progression-free survival following radical cystectomy for urothelial carcinoma. World J Urol. 2014;32:385-391.

20. Boorjian SA, Kim SP, Tollefson MK, et al. Comparative performance of comorbidity indices for estimating perioperative and 5-year all cause mortality following radical cystectomy for bladder cancer. $J$ Urol. 2013;190:55-60.

21. Mayr R, May M, Martini T, et al. Comorbidity and performance indices as predictors of cancer-independent mortality but not of cancer-specific mortality after radical cystectomy for urothelial carcinoma of the bladder. Eur Urol. 2012;62:662-670.

22. American Urological Association. Best practice policy statement on urologic surgery antimicrobial prophylaxis. 2008. Available from: http:// www.auanet.org/education/guidelines/antimicrobial-prophylaxis.cfm. Accessed August 4, 2015.

23. Zaid HB, Kaffenberger SD, Chang SS. Improvements in safety and recovery following cystectomy: reassessing the role of pre-operative bowel preparation and interventions to speed recovery of post-operative bowel function. Curr Urol Rep. 2013;14:78-83.

24. Bochner BH, Dalbagni G, Sjoberg DD, et al. Comparing open radical cystectomy and robot-assisted laparoscopic radical cystectomy: a randomized clinical trial. Eur Urol. 2015;67:1042-1050.

25. Yuh B, Wilson T, Bochner B, et al. Systematic review and cumulative analysis of oncologic and functional outcomes after robot-assisted radical cystectomy. Eur Urol. 2015;67:402-422.

26. Leow JJ, Reese SW, Jiang W, et al. Propensity-matched comparison of morbidity and costs of open and robotic-assisted radical cystectomies: a contemporary population-based analysis in the United States. Eur Urol. 2014;66:569-576.

27. Nguyen DP, Al Hussein AA, Wu X, et al. Recurrence patterns after open and robot-assisted radical cystectomy for bladder cancer. Eur Urol. Epub 2015 Feb 20.

28. Ahmed K, Khan SA, Hayn MH, et al. Analysis of intracorporeal compared with extracorporeal urinary diversion after robot-assisted radical cystectomy: results from the International Robotic Cystectomy Consortium. Eur Urol. 2014;65:340-347.

29. Zehnder P, Studer UE, Skinner EC, et al. Super extended versus extended pelvic lymph node dissection in patients undergoing radical cystectomy for bladder cancer: a comparative study. J Urol. 2011;186:1261-1268.
30. Morgan TM, Kaffenberger SD, Cookson MS. Surgical and chemotherapeutic management of regional lymph nodes in bladder cancer. $J$ Urol. 2012;188:1081-1088.

31. Morgan TM, Barocas DA, Penson DF, et al. Lymph node yield at radical cystectomy predicts mortality in node-negative and not node-positive patients. Urology. 2012;80:632-640.

32. Tilki D, Brausi M, Colombo R, et al. Lymphadenectomy for bladder cancer at the time of radical cystectomy. Eur Urol. 2013;64:266-276.

33. Lee CT, Chang SS, Kamat AM, et al. Alvimopan accelerates gastrointestinal recovery after radical cystectomy: a multicenter randomized placebo-controlled trial. Eur Urol. 2014;66:265-272.

34. Kauf TL, Svatek RS, Amiel G, et al. Alvimopan, a peripherally acting $\mu$-opioid receptor antagonist, is associated with reduced costs after radical cystectomy: economic analysis of a phase 4 randomized, controlled trial. J Urol. 2014;191:1721-1727.

35. Daneshmand S, Ahmadi H, Schuckman AK, et al. Enhanced recovery protocol after radical cystectomy for bladder cancer. J Urol. 2014;192: $50-55$.

36. American Urological Association. Best practice statement for the prevention of deep vein thrombosis in patients undergoing urologic surgery. 2008. Available from: https://www.auanet.org/common/pdf/ education/clinical-guidance/Deep-Vein-Thrombosis.pdf. Accessed August 4, 2015.

37. Sun AJ, Djaladat H, Schuckman A, Miranda G, Cai J, Daneshmand S. Venous thromboembolism following radical cystectomy: significant predictors, comparison of different anticoagulants, and timing of events. J Urol. 2014;193:565-569.

38. von der Maase H, Hansen SW, Roberts JT, et al. Gemcitabine and cisplatin versus methotrexate, vinblastine, doxorubicin, and cisplatin in advanced or metastatic bladder cancer: results of a large randomized, multinational, multicenter, phase III study. J Clin Oncol. 2000;17:3068-3077.

39. International Collaboration of Trialists, Medical Research Council Advanced Bladder Cancer Working Party, European Organisation for Research and Treatment of Cancer Genito-Urinary Tract Cancer Group, et al. International phase III trial assessing neoadjuvant cisplatin, methotrexate, and vinblastine chemotherapy for muscle- invasive bladder cancer: long-term results of the BA06 30894 trial. J Clin Oncol. 2011;29:2171-2177.

40. Sternberg CN, Bellmunt J, Sonpavde G, et al. ICUD-EUA International Consultation on Bladder Cancer 2012: chemotherapy for urothelial carcinoma - neoadjuvant and adjuvant settings. Eur Urol. 2013;63:58-66.

41. Petrelli F, Coinu A, Cabiddu M, Ghilardi M, Vavassori I, Barni S. Correlation of pathologic complete response with survival after neoadjuvant chemotherapy in bladder cancer treated with cystectomy: a meta-analysis. Eur Urol. 2014;65:350-357.

42. Advanced Bladder Cancer (ABC) Meta-analysis Collaboration. Neoadjuvant chemotherapy in invasive bladder cancer: update of a systematic review and meta-analysis of individual patient data: Advanced Bladder Cancer (ABC) Meta-analysis Collaboration. Eur Urol. 2005;48: 202-205.

43. David KA, Mikowsky MI, Ritchey J, Carroll PR, Nanus DM. Low incidence of perioperative chemotherapy for stage III bladder cancer from 1998 to 2003: a report from the National Cancer Data Base. J Urol. 2007;178:451-454.

44. Galsky MD, Pal SK, Chowdhury S, et al. Comparative effectiveness of gemcitabine plus cisplatin versus methotrexate, vinblastine, doxorubicin, plus cisplatin as neoadjuvant therapy for muscle-invasive bladder cancer. Cancer. 2015;121:2586-2593.

45. Sternberg CN, de Mulder P, Schornagel JH, et al. Seven year update of an EORTC phase III trial of high-dose intensity MVAC chemotherapy and G-CSF versus classic M-VAC in advanced urothelial tract tumours. Eur J Cancer. 2006;42:50-54.

46. Lucca I, Roupret M, Kluth L, et al. Adjuvant cisplatin-based combination chemotherapy for lymph node positive urothelial carcinoma of the bladder following radical cystectomy: a retrospective international study of more than 1500 patients. BJU Int. 2015;115:722-727. 
47. Sternberg CN, Skoneczna I, Kerst JM, et al. Immediate versus deferred chemotherapy after radical cystectomy in patients with pT3-pT4 or N+ M0 urothelial carcinoma of the bladder (EORTC 30994): an intergroup, open-label, randomised phase 3 trial. Lancet Oncol. 2015;16:76-86.

48. Arcangeli G, Arcangeli S, Strigari L. A systematic review and metaanalysis of clinical trials of bladder-sparing trimodality treatment of muscle-invasive bladder cancer (MIBC). Crit Rev Oncol Hematol. 2015;94:105-115.

49. Mak RH, Hunt D, Shipley WU, et al. Long-term outcomes in patients with muscle-invasive bladder cancer after selective bladder-preserving combined-modality therapy: a pooled analysis of Radiation Therapy Oncology Group protocols 8802, 8903, 9506, 9906, and 0233. J Clin Oncol. 2014;32:3801-3809.
50. James ND, Hussain SA, Hall E, et al. Radiotherapy with or without chemotherapy in muscle-invasive bladder cancer. $N$ Engl J Med. 2012;366:1477-1488.

51. Miyata Y, Nomata K, Ohba K, et al. Efficacy and safety of systemic chemotherapy and intra-arterial chemotherapy with or without radiotherapy for bladder preservation or as neo-adjuvant therapy in patients with muscle-invasive bladder cancer: a single-centre study of 163 patients. Eur J Surg Oncol. 2015;41:361-367.

52. Azuma H, Inamoto $\mathrm{T}$, Takahara $\mathrm{K}$, et al. Novel bladder preservation therapy with Osaka Medical College regimen. J Urol. 2015;193: 443-450.

53. Siegel R, Ma J, Zou Z, Jemal A. Cancer statistics, 2014. CA Cancer J Clin. 2014;64:9-29.

\section{Publish your work in this journal}

Research and Reports in Urology is an international, peer-reviewed, open access journal publishing original research, reports, editorials, reviews and commentaries on all aspects of adult and pediatric urology in the clinic and laboratory including the following topics: Pathology, pathophysiology of urological disease; Investigation and treatment of

\section{Dovepress}

urological disease; Pharmacology of drugs used for the treatment of urological disease. The manuscript management system is completely online and includes a very quick and fair peer-review system, which is all easy to use. Visit http://www.dovepress.com/testimonials.php to read real quotes from published authors.

Submit your manuscript here: http://www.dovepress.com/research-and-reports-in-urology-journal 